Artículo

\title{
Ordenación de la vegetación de las sierras y llanuras occidentales municipio de Catorce, San Luis Potosí
}

\author{
Cándido Díaz-Reyes ${ }^{1}$ \\ Diodoro Granados-Sánchez ${ }^{1}$ \\ Miguel Uribe-Gómez ${ }^{1}$ \\ Dante Arturo Rodríguez-Trejo ${ }^{1 \S}$ \\ Ro Linx Granados Victorino ${ }^{2}$
}

${ }^{1}$ Maestría en Agroforestería para el Desarrollo Sostenible-Universidad Autónoma Chapingo. Carretera México-Texcoco km 38.5, Chapingo, Estado de México, CP. 56230. (diaz.candido.005@gmail.com; diodorog@hotmail.com; migueluribe123@gmail.com). ${ }^{2}$ Preparatoria Agrícola-Universidad Autónoma Chapingo.

${ }^{\S}$ Autor para correspondencia: dantearturo@yahoo.com.

\section{Resumen}

La ordenación de comunidades vegetales puede usar métodos multivariados para hallar patrones de respuesta y generar hipótesis sobre las posibles relaciones existentes en la composición de especies con respecto a gradientes ambientales. El objetivo fue registrar patrones de distribución y usos de la vegetación en el municipio de Catorce, San Luis Potosí. En 2019, se muestrearon seis transectos, desde pie de monte bajo al oeste de la Sierra de Catorce, valle o planicie de drenaje endorreico y sierra baja plegada con lomerío. En cada sitio se analizó la composición florística, la estructura vertical y horizontal y las características del suelo. Se hallaron matorrales desérticos rosetófilo, micrófilo y bosque espinoso. Los factores ambientales indirectos que mejor explican la distribución son la porosidad del suelo, $\mathrm{pH}, \mathrm{K}, \mathrm{Ca}, \mathrm{Fe}$ y altitud, factores que influyen en la disponibilidad de agua. El principal uso de las plantas es medicinal tradicional.

Palabras clave: sinecología, Wirikuta, zonas áridas.

Recibido: marzo de 2020

Aceptado: mayo de 2020 


\section{Introducción}

Desierto es aquella superficie de tierra cuyos potenciales biológicos están severamente limitados por la escasez de agua, con precipitaciones $<250 \mathrm{~mm}$ anuales y temperaturas generalmente elevadas (Granados, 2012). La disponibilidad de agua y los factores físicos relacionados con ésta son primordiales y explican la variación en los patrones de distribución de la vegetación en estos, Schlesinger (1998); Pavón (2000) sostiene que la distribución de arbustos en hábitats desérticos está fuertemente correlacionada con la presencia de N, P y K en el suelo, debido a que bajo su dosel se concentra el ciclo biogeoquímico de estos elementos en 'islas de fertilidad'.

La región árida más grande del continente americano es el Desierto Chihuahuense (DC), ecorregión ubicada en una planicie triangular, al norte de México entre la Sierra Madre Oriental, la Sierra Madre Occidental y el Eje Neovolcánico, la cual se prolonga hacia Nuevo México y Texas. Se estima que en el DC existen 324 especies de cactáceas, 30\% de las del planeta, la mayoría endémicas (Rzedowski, 1994), reconociéndose a San Luis Potosí (SLP) como el estado con mayor diversidad de especies, con 151 del total de 826 taxones registrados en el DC, 560 (67.8\%) son endémicos, 165 (20\%) cuasi-endémicos y 176 (21.6\%) micro-endémicos (Villarreal, 2017).

Con 17 géneros de cactus (43.6\%) y 229 especies (70\%) restringidas al DC, la tasa de endemismo en esta región es sobresaliente (Hernández, 2004). Las sierras y llanuras occidentales del municipio de catorce, son una zona árida de gran interés para estudios de vegetación, debido a su composición de especies endémicas, de distribución restringida y en peligro de extinción; también su topografía, edafología y el deterioro permanente que han sufrido, son tema de peculiar interés en esta área natural protegida (ANP) bajo el estatus de 'sitio sagrado natural' que alberga una herencia biocultural excepcional.

El presente proyecto es una primera etapa que busca obtener información sinecológica de base para el diseño de un sistema agroforestal medicinal tradicional en una segunda etapa. El objetivo de esta investigación fue emplear técnicas multivariadas para identificar patrones de distribución de la vegetación a lo largo de gradientes ambientales en los valles y llanuras occidentales del municipio de Catorce, San Luis Potosí (SLP).

\section{Materiales y métodos}

La investigación se realizó en la subprovincia de las sierras y llanuras occidentales, municipio de Catorce, SLP. Forma parte de la provincia fisiográfica Mesa del Centro, al oeste de la Sierra de Catorce (SC), en el cuadrante SE de la ecorregión del DC, al sureste del altiplano potosinozacatecano, en la porción NO de SLP, en valles y llanuras intermontanos de drenaje endorreico formados principalmente por grava, arcillas y arenas, que forman estructuras de acumulación forzada con los acarreos fluviales de materiales de la SC. Los tipos de clima en el sitio de estudio son árido-templado (BSokw) y muy árido-templado (BWkw).

Los suelos predominantes en las planicies situadas en los flancos este y oeste de la SC son xerosoles, suelos caracterizados por su capa superficial de material claro con bajo contenido de materia orgánica (CONANP, 2012). Se realizaron recorridos de campo para determinar los tipos de vegetación; a través de un gradiente altitudinal (1 600-1 910 m), se establecieron tres áreas de muestreo en función de las asociaciones vegetales: el matorral desértico microfilo (MDM) en el 
valle, matorral desértico rosetófilo (MDR) en sierra baja y bosque abierto de mezquite o bosque espinoso (BE) a pie de monte de la SC. Se elaboró un mapa de tipos de vegetación (Figura 1) del municipio de Catorce, en el que se señalan los tres sitios de muestreo elegidos, para lo cual se empleó el programa ArcGis 10.3.
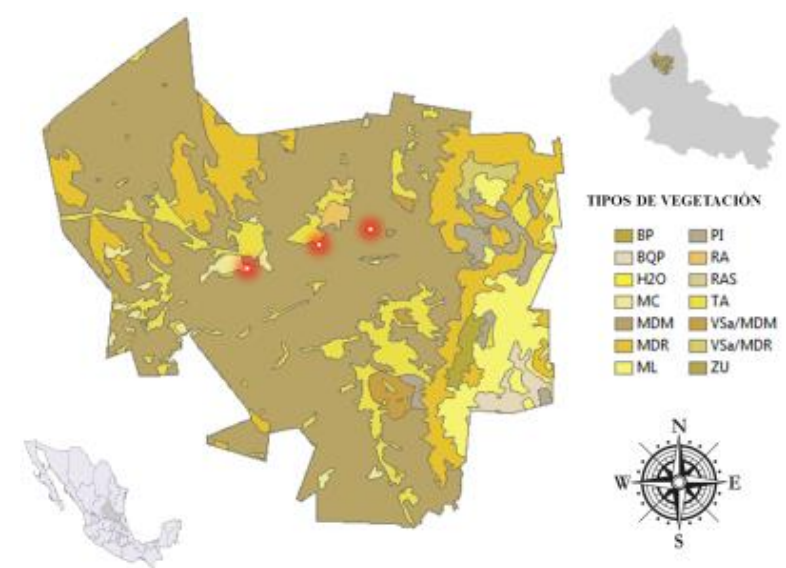

Figura 1. Tipos de vegetación y sitios de muestreados en el municipio de Catorce, SLP.

Se levantaron seis transectos de muestreo, por cada tipo de comunidad observada, paralelos, con una distancia de $3 \mathrm{~km}$ entre sí. El método de muestreo empleado para caracterizar cuantitativamente las comunidades vegetales fue punto central y cuadrantes (Brush, 1980), con 10 puntos de muestreo (60 puntos en total y 240 cuadrantes). Con este muestreo se obtuvieron la dominancia, densidad y frecuencia, tanto absoluta como relativa y valor de importancia relativa (VIR).

En cada punto de muestreo, también se registró la composición florística (perennes y anuales), en un sitio de 10 x10 m (60 sitios). Las plantas que no pudieron ser identificadas en campo fueron recolectadas y prensadas para su posterior identificación en el herbario de Preparatoria Agrícola, Universidad Autónoma Chapingo (UACH). En los sitios también se obtuvieron muestras compuestas de suelo $(1 \mathrm{~kg}$ ) de cada una de las asociaciones vegetales (MDM, MDR y BE).

Las muestras fueron enviadas al Laboratorio Central de Suelos de la Universidad Autónoma Chapingo (UACH) para la determinación de: $\mathrm{pH}$ (potenciométrico), $\mathrm{N}$ inorgánico (extraído con cloruro de potasio $2 \mathrm{~N}$ y determinado por arrastre de vapor), $\mathrm{P}$ asimilable (Olsen), $\mathrm{K}$ (extraído en acetato de amonio $1 \mathrm{~N}, \mathrm{pH}$ 7, relación 1:20 y determinado por espectrofotometría de emisión de flama), Fe (extraído con DTPA en una relación 1:4 y determinado por espectrofotometría de absorción atómica), $\mathrm{Ca}$ (extraído en acetato de amonio 1 N, pH 7, relación 1:20

Determinado por espectrofotometría de absorción atómica), textura (hidrómetro de Bouyoucos). La ordenación de la vegetación fue de dos tipos: 1) ordenación indirecta mediante el análisis de agrupamiento (cluster), construyendo las agrupaciones en conjuntos de alta similitud interna o disimilitud, tomando en cuenta los miembros de otros grupos. La estructura de la clasificación obtenida de la matriz de similitud se representó en un dendrograma (Rocha, 2006). Para la clasificación numérica de la vegetación por medio de agrupamientos basados en medidas de distancia y métodos de unión de grupos se empleó el programa SPSS V23.0. 
Esta clasificación se realizó a partir de variables cualitativas, con base en atributos binarios (presencia ausencia) y fue tipo politética aglomerativa. Se incluyeron un total de 78 especies. Los grupos fueron formados empleando el índice de Jaccard como medida de distancia y como algoritmo de unión el promedio entre grupos; y 2) ordenación directa mediante análisis de correspondencia canónica (ACC), la cual es una técnica que representa un caso especial de regresión múltiple, donde la composición de las especies es directamente relacionada con las variables ambientales (Gonzáles, 2003), para realizar el análisis se utilizó el programa de cálculo CANOCO y se elaboró su representación gráfica empleando el programa CANODRAW (Braak, 1998). Se estimó el valor de importancia relativa de las especies y se elaboró un perfil fisionómico para describir las comunidades; se empleó el programa Lumion.

La descripción de las comunidades se realizó en base a la literatura (Granados, 2012; GonzálezCostilla, 2007; CONANP, 2012) y en la observación en recorridos de campo con actores clave (productores, recolectores, vendedores de planta medicinal, botánicos y especialistas en plantas medicinales), que también fueron entrevistados sobre el uso de las plantas, particularmente el medicinal.

\section{Resultados y discusión}

\section{Análisis de correspondencia}

La ordenación indirecta o análisis de correspondencia (AC) de las especies en los seis transectos muestreados se presenta en un dendrograma (Figura 2). Se aprecian de forma general tres asociaciones vegetales eligiendo como nivel de corte $41 \%$ de la información (media). La primera asociación es de MDM (transectos 1 y 2), ubicado en el valle entre la SC y sierra baja, a 1600 msnm al norte de la comunidad.

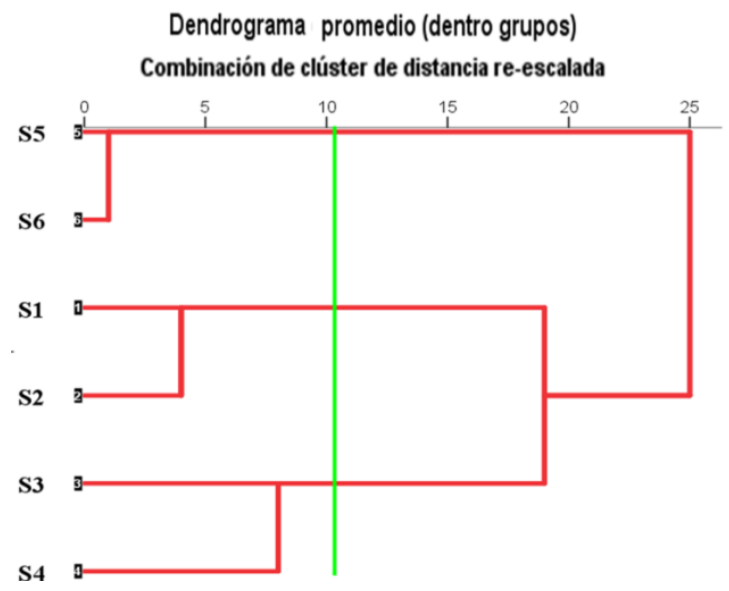

Figura 2. Dendrograma que muestra relaciones jerárquicas entre transectos muestreados.

La Borrega. El segundo tipo de vegetación (transectos 5 y 6) es el BE, ubicado a pie de monte de la SC, a 1770 msnm, en la comunidad de San Cristóbal. El tercer grupo (transectos 3 y 4) es el MDR, distribuido en sierra baja con lomerío, a 1910 msnm al NE de la comunidad El Tecolote. 
En el AC se reduce el espacio multidimensional, conservando las distancias entre los objetos (muestras de vegetación), posibilitando determinar la correspondencia entre las columnas (transectos de muestreo) y las filas (especies) de la matriz de datos (de la Cruz, 1991). El Cuadro 1 presenta los valores de proximidad o correspondencia entre las especies y transectos de muestreo para los primeros ejes de ordenación.

Cuadro 1. Valores de correspondencia entre especies y transectos para los ejes de ordenación.

\begin{tabular}{ccccccc}
\hline \multicolumn{7}{c}{ Matriz de proximidades } \\
\hline Objetos & S1 & S2 & S3 & S4 & S5 & S6 \\
\hline S1 & & & & & & 239 \\
S2 & 923 & & & & & 250 \\
S3 & 400 & 443 & & & & 277 \\
S4 & 412 & 456 & 821 & & & 246 \\
S5 & 239 & 250 & 277 & 246 & & 1 \\
S6 & 239 & 250 & 277 & 246 & 0.996 & 1 \\
\hline
\end{tabular}

En la Figura 3 es posible visualizar que los transectos de muestreo 1 y 2 están ubicados muy cerca entre sí, esto significa que los transectos comparten gran número de especies, de manera similar, los transectos de muestreo 3 y 4 tienen una proximidad reducida, así como los transectos 5 y 6.

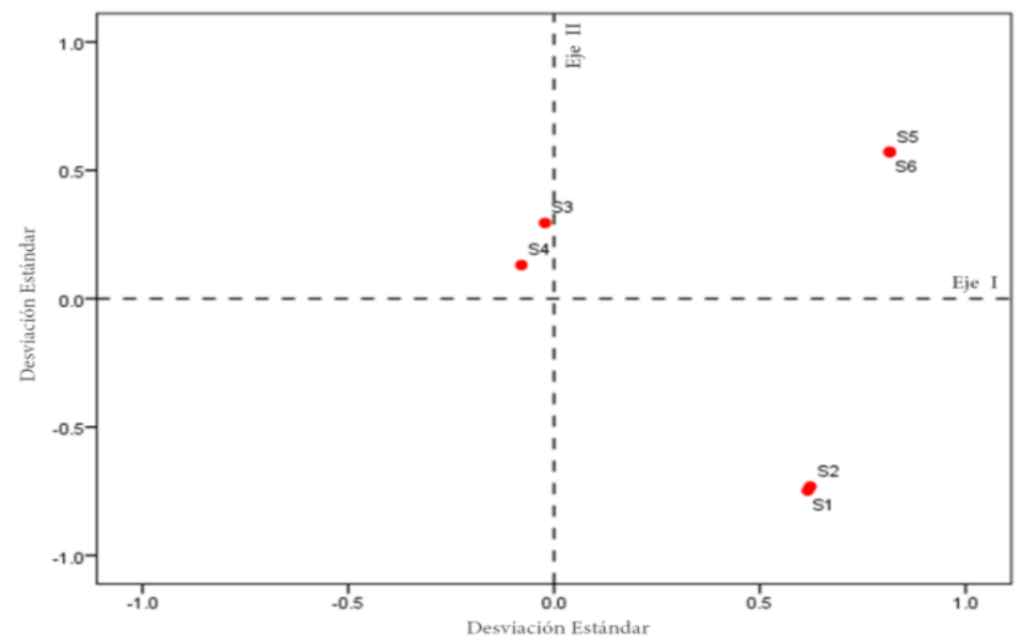

Figura 3. Diagrama de ordenación indirecta, ubicación de transectos de muestreo en el espacio de ordenación.

\section{Matorral desértico micrófilo, comunidad La Borrega}

El MDM de este sitio se ubica $10 \mathrm{~km}$ al O de la SC en la subprovincia de las sierras y llanuras occidentales, en un valle de drenaje endorreico, compuesto por material de acarreo, principalmente grava, arcillas y arenas. Se localiza en las coordenadas $23^{\circ} 43^{\prime} 52.5^{\prime \prime}$ latitud norte y $101^{\circ} 04^{\prime} 46.0^{\prime}$ ' longitud oeste, a $1600 \mathrm{msnm}$, presenta una PMA de $311 \mathrm{~mm}$, el clima es árido-templado (BSokw), con verano cálido, TMA de 12 a $18{ }^{\circ} \mathrm{C}$, la del mes más frío entre -3 y $18{ }^{\circ} \mathrm{C}$ y la del mes más cálido $>18 \mathrm{C}$, lluvias de verano, de 5 a $10 \%$ anual. 
Los suelos tienen las siguientes características: $\mathrm{pH}$ (7.78), $\mathrm{N}\left(3.8 \mathrm{mg} \mathrm{kg}^{-1}\right), \mathrm{P}\left(22.81 \mathrm{mg} \mathrm{kg}^{-1}\right), \mathrm{K}$ (490 mg kg-1), Ca $\left(6721 \mathrm{mg} \mathrm{kg}^{-1}\right), \mathrm{Fe}\left(2.37 \mathrm{mg} \mathrm{kg}^{-1}\right)$. El suelo es de textura franco-arenosa $(54.4 \%$ arena, $29.3 \%$ limo y $16.4 \%$ arcilla). El contenido de materia orgánica y la textura del suelo obtenidas coinciden con los resultados de la CONANP (2012), en el cual se indica que esta planicie (con suelos xerosoles) se caracteriza por presentar una capa superficial de material claro, debida al bajo contenido de materia orgánica.

En una evaluación a lo largo de un transecto permanente (3 años), que atraviesa una cuenca endorreica en la porción N del DC, en el centro-sur de Nuevo México, se determinó que el estrato arbustivo dominado por Larrea tridentata D. Burckhardt, se extendía en la porción xérica, muy baja en nitrógeno; las hierbas perennes con senda fotosintética $\mathrm{C} 4$, los arbustos con senda $\mathrm{C} 3$ y las herbáceas fueron codominantes en los sitios intermedios a lo largo del gradiente (Cornelius, 1991).

Este matorral se caracteriza por la dominancia de especies arbustivas de hojas pequeñas, plantas crasas y efímeras que se desarrollan a lo largo de abanicos aluviales, planicies, bajadas, valles y lomeríos suaves, su principal distribución son los valles, donde los suelos son de textura fina, profundos a relativamente profundos y presentan una capa de rocas (Granados, 2012).

En este matorral se identificaron 28 especies distribuidas en 12 familias, de las cuales Cactaceae y Asteraceae fueron las más representativas, con 9 y 6 especies, respectivamente (Cuadro 1) del material suplementario. Seis especies son endémicas, una está amenazada y dos bajo protección especial.

Se halló una riqueza de cactáceas de 10 especies, destacan Cylindropuntia imbricata (Haw.) F. M. Knuth, Cylindropuntia leptocaulis J. Jones, Echinocactus horizonthalonius J. Daniel, Echinocactus platyacanthus R. F. del Castillo, Echinocereus enneacanthus D. Sánchez, Echinocereus pectinatus N. P. Taylor, Escobaria dasyacantha D. R. Hunt, Ferocactus hamatacanthus J. Y. Clark, F. latispinus J. H. Cota, F. pilosus N. P. Taylor, F. stainesii R. S. Wallace, Lophophora williamsii E. F. Anderson, Opuntia rastrera J. A. Reyes-Agüero y Opuntia tunicata A. C. Gibson.

El análisis de valor de importancia relativa (VIR) se presenta en el Cuadro 2, donde se aprecian las especies con VIR más alto: L. tridentata, seguida por C. leptocaulis, Jatropha dioica B. Dehgan, O. tunicata, F. cernua M. O. Dillon y E. enneacanthus. Una foto de la comunidad y un perfil fisonómico semirrealista, se muestran en la Figura 4a y 4b).

Cuadro 2. Valores de importancia para las especies del MDM, La Borrega, municipio Catorce.

\begin{tabular}{ccccccccc}
\hline Especie & $\mathrm{ABM}^{\mathrm{Z}}$ & $\mathrm{AA}^{\mathrm{Y}}$ & $\mathrm{FA}^{\mathrm{X}}$ & $\mathrm{DA}^{\mathrm{W}}$ & $\mathrm{AR}^{\mathrm{V}}$ & $\mathrm{FR}^{\mathrm{U}}$ & $\mathrm{DR}^{\mathrm{T}}$ & $\mathrm{VIR}^{\mathrm{S}}$ \\
\hline Larrea tridentata & 10.9 & 29 & 26 & 50 & 24.2 & 22.6 & 22.6 & 23.1 \\
Cylindropuntia leptocaulis & 4.4 & 19 & 14 & 29.9 & 15.8 & 13.2 & 6 & 11.7 \\
Jatropha dioica & 8.5 & 10 & 7 & 15.8 & 8.3 & 6.6 & 6.1 & 7 \\
Opuntia tunicata & 23.3 & 6 & 6 & 12.1 & 5 & 5.7 & 10 & 6.9 \\
Flourensia cernua & 10 & 8 & 8 & 15 & 6.7 & 7.6 & 5.7 & 6.6 \\
Echinocereus enneacanthus & 25.2 & 5 & 5 & 10.1 & 4.2 & 4.7 & 9 & 6 \\
\hline
\end{tabular}

$\mathrm{Z}_{\text {}}$ área basal media; ${ }^{\mathrm{Y}}=$ abundancia absoluta; ${ }^{\mathrm{X}}=$ frecuencia absoluta; ${ }^{\mathrm{W}}=$ dominancia absoluta; ${ }^{\mathrm{V}}=$ abundancia relativa;

$\mathrm{U}=$ frecuencia relativa; ${ }^{\mathrm{T}}=$ dominancia relativa; ${ }^{\mathrm{S}}=$ valor de importancia relativa. 
a)

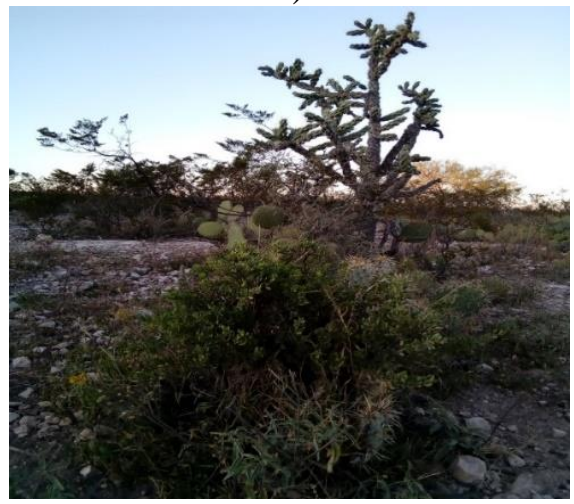

c)

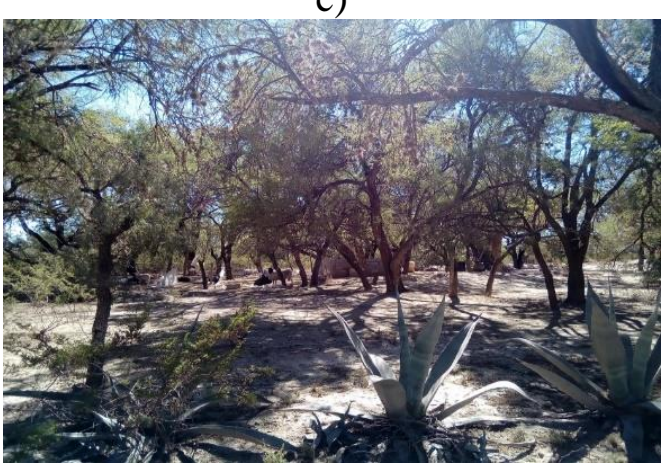

e)

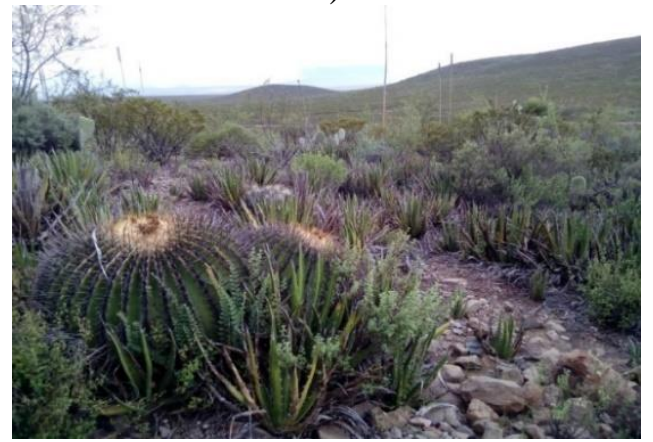

b)

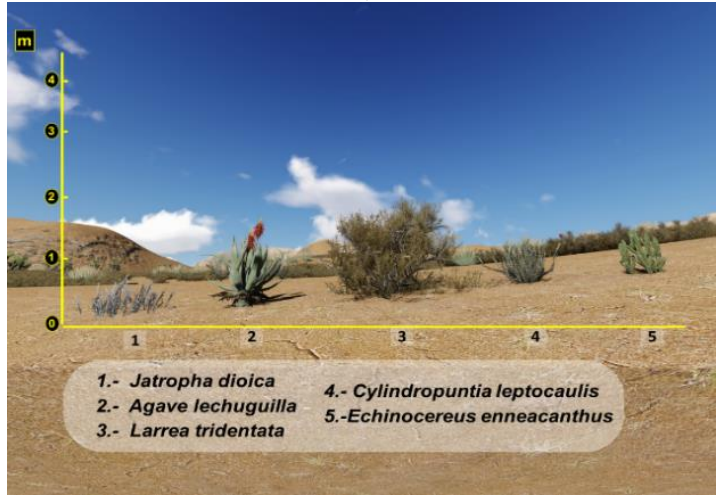

d)

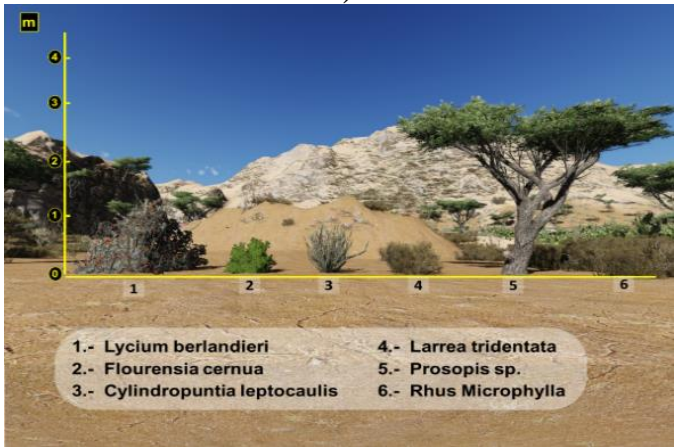

f)

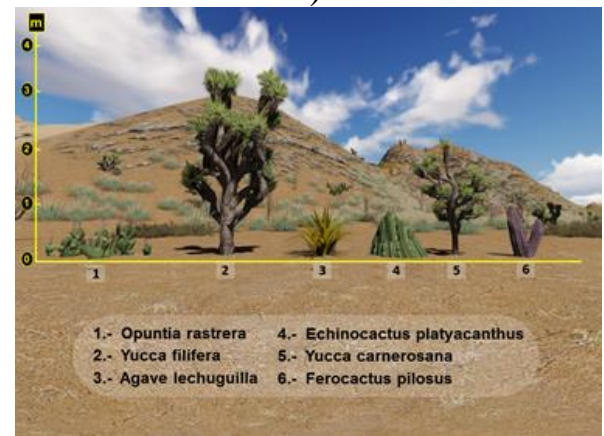

Figura 4. a) especies del MDM en la comunidad La Borrega; b) su perfil semirrealista; c) Mezquitales del municipio de Catorce; d) su perfil semirrealista, comunidad San Cristóbal; e) MDR, comunidad El Tecolote, municipio de Catorce; y f) Perfil semirrealista del MDR.

Los resultados obtenidos son análogos a la descripción que se realiza en el análisis de la flora vascular, realizado en la SC y territorios adyacentes (González-Costilla, 2007), en el cual se describe a esta área como una formación dominada por las especies Larrea tridentata, Parthenium incanum G. K. Brizicky, Zinnia acerosa A. M. Torres, Flourensia cernua, Opuntia imbricata, Opuntia leptocaulis, Prosopis laevigata M. C. Johnston y Fouquieria splendens M. Baker. De acuerdo con Marroquín (1964), el estrato dominante en este tipo de matorral es el estrato arbustivo e incluye con frecuencia a L. tridentata y F. cernua. 


\section{Bosque espinoso, comunidad San Cristóbal}

El BE se localiza en el lindero de las provincias fisiográficas Sierra Madre Oriental y Mesa del Centro. Ubicado a pie de monte bajo, $6 \mathrm{~km}$ al oeste de la SC, en valles y llanuras intermontanas de drenaje endorreico, conformados por estructuras de acumulación forzada. Ahí suele presentarse la denominada playa de sustrato arenoso a $1770 \mathrm{msnm}$. La TMA es $16.6^{\circ} \mathrm{C}$, con una máxima de 37 ${ }^{\circ} \mathrm{C}$ y una mínima de $-7^{\circ} \mathrm{C}$. La PMA es de $311 \mathrm{~mm}$.

El clima se considera templado-seco ( $\mathrm{BS}_{0} \mathrm{hx}$ '), el más seco de los $\mathrm{BS}$, semicálido con régimen de lluvias intermedio, invierno fresco y con precipitación invernal mayor a 18\% (SEDUVOP, 2006). Esta área es catalogada como una superficie fértil y con potencial agrícola, con disponibilidad de agua subterránea, cuya salinidad es alta y bajo contenido de sodio. Sin embargo, también es clasificado como un sitio abierto de mezquite con un grado de erosión alto, por la acción del viento, y suelo arenoso. Se halla en el límite norte del ANP, por lo que su expansión agrícola compromete la conservación del sitio.

El suelo tiene las siguientes características: $\mathrm{pH}$ (7.31), $\mathrm{N}\left(26.3 \mathrm{mg} \mathrm{kg}^{-1}\right), \mathrm{P}\left(31.9 \mathrm{mg} \mathrm{kg}^{-1}\right), \mathrm{K}(2240$ $\left.\mathrm{mg} \mathrm{kg}{ }^{-1}\right), \mathrm{Ca}\left(6206 \mathrm{mg} \mathrm{kg}^{-1}\right), \mathrm{Fe}\left(3.4 \mathrm{mg} \mathrm{kg}^{-1}\right)$. El suelo es de textura franca (46.4\% arena, 39.3\% limo y $14.4 \%$ arcilla). El alto contenido de P, K, y MO del suelo de este sitio (en comparación con el MDM) se debe quizás a la presencia de Prosopis sp., ya que estudios en zonas áridas demuestran que el mezquite aumenta $\mathrm{N}$ y materia orgánica en el suelo (Herrera-Arreola et al., 2007).

Autores como Reyes-Reyes (2003) reporta que la mineralización de C en las hojas de mezquite es mayor que en las hojas de huizache, también demuestra que la liberación de $\mathrm{N}$ de las hojas es mayor cuando el contenido de materia orgánica del suelo es menor, bajo el dosel del mezquite se acumula materia orgánica, microorganismos y nutrientes. Se identificaron 22 especies de 11 familias; Asraceae y Fabaceae fueron las más representativas (Cuadro 2) del material suplementario.

También se hallaron dos especies endémicas. En esta área, la presencia de especies arbustivas de hojas pequeñas y plantas efímeras características del MDM es frecuente. No obstante, este sitio se destaca por la presencia de especies freatófilas arbustivas y arbóreas, principalmente Prosopis L. y Acacia farnesiana (L.) Willd. Su principal distribución está influenciada por cuerpos de agua subterráneos, por lo que su presencia en pie de monte es abundante, se desarrolla a lo largo de abanicos aluviales en los que comparte la dominancia con Lycium berlandieri (T. Fukuda), $L$. tridentata, F. cernua y Rhus microphylla (J. S. Lassetter).

La riqueza de cactáceas en este sitio es baja, únicamente se encontró a Cylindropuntia leptocaulis, también presente en MDM y MDR. Las especies asociadas en este sitio fueron: Ephedra compacta, Bouteloua scorpioides J. T. Columbus, Artemisia filifolia E. D. McArthur, Bidens odorata W. C. Yang, Zinnia acerosa, Artemisia ludoviciana E. D. McArthur, Flourensia cernua, Gymnosperma glutinosum J. Ruffin, Koeberlinia spinosa W. C. Holmes y Larrea tridentata.

El número de especies en esta área es la más baja de los tres sitios, similar al estudio realizado en el sur del DC (Montaña, 1990), con la menor riqueza de especies en la playa de sustrato arenoso. En el análisis de valor de importancia relativa (VIR) (Cuadro 3), se aprecian las especies con VIR más alto: Lycium berlandieri, seguida por Prosopis sp., L. tridentata, F. cernua, R. microphylla y C. leptocaulis. 
Cuadro 3. VIR para las especies del BE, comunidad San Cristóbal, municipio de Catorce.

\begin{tabular}{ccccccccc}
\hline Especie & $\mathrm{ABM}^{\mathrm{Z}}$ & $\mathrm{AA}^{\mathrm{Y}}$ & $\mathrm{FA}^{\mathrm{X}}$ & $\mathrm{DA}^{\mathrm{W}}$ & $\mathrm{AR}^{\mathrm{V}}$ & $\mathrm{FR}^{\mathrm{U}}$ & $\mathrm{DR}^{\mathrm{T}}$ & $\mathrm{VIR}^{\mathrm{S}}$ \\
\hline Lycium berlandieri & 5.6 & 14 & 14 & 0.78 & 11.7 & 11.8 & 9.4 & 10.9 \\
Prosopis sp. & 16.6 & 8 & 8 & 1.33 & 6.7 & 6.7 & 16.02 & 9.8 \\
Larrea tridentata & 5.1 & 13 & 12 & 0.66 & 10.8 & 10.1 & 7.95 & 9.6 \\
Flourensia cernua & 7.6 & 11 & 10 & 0.84 & 9.2 & 8.4 & 10.12 & 9.2 \\
Rhus Microphylla & 6.3 & 12 & 10 & 0.75 & 10 & 8.4 & 9.04 & 9.1 \\
Cylindropuntia leptocaulis & 4.3 & 12 & 12 & 0.52 & 10 & 10.1 & 6.27 & 8.8 \\
\hline
\end{tabular}

$\mathrm{Z}_{\mathrm{Z}}$ área basal media; ${ }^{\mathrm{Y}}=$ abundancia absoluta; $^{\mathrm{X}}=$ frecuencia absoluta $;{ }^{\mathrm{W}}=$ dominancia absoluta; ${ }^{\mathrm{V}}=$ abundancia relativa;

$\mathrm{U}=$ frecuencia relativa; ${ }^{\mathrm{T}}=$ dominancia relativa; ${ }^{\mathrm{S}}=$ valor de importancia relativa.

La descripción de Rzedowski (1994) del MDM del DC, es similar a estos resultados, con la eventual aparición de comunidades de Prosopis sp., Koeberlinia spinosa o Fouquieria splendens, que se extienden por cientos de kilómetros en el DC Granados (2012) refiere arbustos salados en sitios abiertos de mezquite, como una comunidad de amplia distribución presente en suelos de textura fina y erosionados por la acción del viento y suelo arenoso; ambas fuentes reportan entre las principales especies a Parthenium incanum, P. argentatum S. Kumar, Viguiera stenoloba E. E. Schilling, Zinnia acerosa, Dyssodia sp. y Jatropha dioica. En la Figura 4c y 4d se muestran foto de este tipo de comunidad y su esquema semirrealista.

\section{Matorral desértico rosetófilo, comunidad El Tecolote}

El MDR de este sitio se ubica $25 \mathrm{~km}$ al O de la SC, en la subprovincia valles y llanuras occidentales ( $23^{\circ} 42^{\prime} 19.2^{\prime}$ ' latitud norte y $101^{\circ} 07^{\prime} 28.8^{\prime}$ " longitud oeste. Se desarrolla a $1910 \mathrm{msnm}$. El clima es muy árido-templado (BWkw), TMA de 12 a $18{ }^{\circ} \mathrm{C}$, la del mes más frío entre $-3^{\circ}$ y $18{ }^{\circ} \mathrm{C}$ y la del mes más cálido $>18{ }^{\circ} \mathrm{C}$, régimen de lluvia de verano de 5 a $10.2 \%$ anual.

El sitio se encuentra en una sierra baja plegada con lomerío en dirección N-S de roca ígnea extrusiva; los suelos son leptosoles (CONANP, 2012). Los suelos presentan las siguientes características: $\mathrm{pH}$ (7.30), $\mathrm{N}\left(57.8 \mathrm{mg} \mathrm{kg}^{-1}\right), \mathrm{P}\left(58.86 \mathrm{mg} \mathrm{kg}^{-1}\right), \mathrm{K}\left(2060 \mathrm{mg} \mathrm{kg}^{-1}\right), \mathrm{Ca}(7814 \mathrm{mg}$ $\left.\mathrm{kg}^{-1}\right)$, Fe (26.84 $\left.\mathrm{mg} \mathrm{kg}^{-1}\right)$. El suelo es de textura franca (42.4\% arena, $35.3 \%$ limo y $22.4 \%$ arcilla). De acuerdo con CONANP (2012), el tipo de suelo en este sitio es característico de unidades geomorfológicas de sistemas montañosos con laderas convexas de cima redondeada de roca calizaarenisca, estas superficies son empleadas para usos pecuarios y de producción de maíz.

De acuerdo con la taxonomía de suelos utilizada el Departamento de Agricultura de Estados Unidos de América, los litosoles de esta área son Entisoles o Aridisoles, suelos con poca evidencia de desarrollo de horizontes pedogénicos y que, en general, sólo presentan un horizonte A ócrico. Se encuentran en pendientes que sufren fuertes procesos erosivos, pueden soportar diferentes tipos de formaciones vegetales y presentan una naturaleza mineral.

El matorral que se desarrolla en este sitio se caracteriza por la abundancia de individuos de especies con hojas gruesas, estrechas y alargadas, en ocasiones espinosas como lechuguilla (Agave lechuguilla H. S. Gentry) o sotol (Dasylirion cedrosanum E. E. Castillón) o bien inermes pero fibrosas (Yucca carnerosana A. M. Minnis), dispuestas en roseta. Entre estos individuos se encuentran básicamente dos tipos de desarrollo: 1). Aquellas especies que despliegan tallo alargado 
y manifiesto cómo (Yucca sp. y Dasylirion sp.); y 2) aquellas que carecen de tallo visible y desarrollan hojas basilares, como (Agave sp. y Hechtia sp.) (Granados, 2012). Grandes valles de lomeríos y sierras bajas albergan en el municipio de Catorce especies como Agave lechuguilla, Yucca filifera E. E. Castillón, Yucca decipiens M. Ricker, Parthenium argentatum y Dasylirion cedrosanum A. Robles-Esparza.

En las pendientes orientales de esta sierra baja (orientación N-S) es frecuente el desarrollo de Opuntia rastrera A. Muñoz-Urias y Opuntia stenopetala A. García-Velázquez, especies como A. lechuguilla, A. striata D. J. Bogler y Hechtia glomerata K. Burt-Utley, se desarrollan en colonias de grandes masas que delinean el paisaje a través de grandes superficies con la eventual aparición de colonias de Fouquieria splendens E. C. Bate-Smith.

Estas especies se desarrollan en el sitio con especies arbustivas y subarbustivas principalmente, las arbóreas son escasas, no hay herbáceas invernales, ni germinación o floración en invierno y la mayoría de las especies son perennifolias. Algunas de las especies más abundantes en este sitio fueron Agave lechuguilla, Euphorbia antisyphilitica E. H. Muldavin, Zinnia acerosa, Parthenium argentatum y Agave striata H. S. Gentry.

En esta comunidad se identificaron 61 especies distribuidas en 16 familias, de las cuales las familias Cactaceae, Asparagaceae y Asteraceae fueron las más representativas (Cuadro 3) del material suplementario. Se hallaron 20 especies endémicas, cuatro amenazadas, seis bajo protección especial y tres en peligro.

La riqueza de cactáceas en este sitio es muy alta, 30 especies, entre las que destacan Cylindropuntia leptocaulis, Cylindropuntia imbricata, Echinocereus stramineus M. Baker, Coryphantha poselgeriana M. A. Baker, Opuntia engelmannii J. G. Jones, O. leucotricha S. Samah, O. stenopetala M. Fuentes-Pérez, Ferocactus pilosus H. M. Hernández y Echinocactus platyacanthus.

La región del DC sobresale por tener la mayor diversidad de cactáceas del planeta, esta familia es la más amenazada en la ecorregión. En todo el DC existen 324 especies de cactus, de 39 géneros. Los géneros Mammillaria, Opuntia, Coryphantha y Echinocereuus, representan 58\% de la diversidad total de la región; 17 (43.6\%) de los 39 géneros de cactus registrados están restringidos al DC. Asimismo, un total de 229 especies (70\%) son endémicas de la región (Hernández, 1994).

Del total de especies registradas en el DC, 826 (67.8\%) son de distribución endémica (Villarreal, 2017). En lo que respecta a la riqueza de cactáceas y endemismo, el estado de SLP es el más importante, debido a que el número total de especies de cactus en 141 (44\% de especies de todo el DC) y el número de especies endémicas del estado es 17 (Hunt, 1999). Por otra parte, Jiménez (2011) reconoce a SLP como el estado con mayor diversidad de especies, con 151 .

El análisis de valor de importancia relativa (VIR) se presenta en el Cuadro 4, donde se aprecian las especies con VIR más alto: Yucca filifera seguida por A. lechuguilla, Y. carnerosana, E. platyacanthus, $F$. pilosus y $O$. rastrera. Foto y perfil semirrealista de esta comunidad, se muestran en la Figura 4e y 4f. 
Cuadro 4. Valores de importancia para las especies del MDR, comunidad EI Tecolote, municipio de Catorce.

\begin{tabular}{ccccccccc}
\hline Especie & $\mathrm{ABM}^{\mathrm{Z}}$ & $\mathrm{AA}^{\mathrm{Y}}$ & $\mathrm{FA}^{\mathrm{X}}$ & $\mathrm{DA}^{\mathrm{W}}$ & $\mathrm{AR}^{\mathrm{V}}$ & $\mathrm{FR}^{\mathrm{U}}$ & $\mathrm{DR}^{\mathrm{T}}$ & $\mathrm{VIR}^{\mathrm{S}}$ \\
\hline Yucca filifera & 48.8 & 12 & 10 & 5.86 & 9.6 & 8.33 & 19.82 & 12.6 \\
Agave lechuguilla & 12.4 & 14 & 13 & 1.73 & 11.2 & 10.83 & 5.85 & 9,6 \\
Yucca carnerosana & 40.3 & 9 & 10 & 3.63 & 7.2 & 8.33 & 12.28 & 9.3 \\
Echinocactus platyacanthus & 44.1 & 7 & 7 & 3.09 & 5.6 & 5 & 10.45 & 7.3 \\
Ferocactus pilosus & 56.5 & 6 & 6 & 3.39 & 4.8 & 5 & 11.46 & 7.1 \\
Opuntia rastrera & 15.3 & 10 & 9 & 1.53 & 8 & 7.5 & 5.27 & 6.9 \\
\hline
\end{tabular}

$\mathrm{Z}_{\mathrm{Z}}$ área basal media; ${ }^{\mathrm{Y}}=$ abundancia absoluta; ${ }^{\mathrm{X}}=$ frecuencia absoluta $;{ }^{\mathrm{W}}=$ dominancia absoluta; ${ }^{\mathrm{V}}=$ abundancia relativa;

$\mathrm{U}=$ frecuencia relativa; ${ }^{\mathrm{T}}=$ dominancia relativa; ${ }^{\mathrm{S}}=$ valor de importancia relativa.

El estrato dominante en el sitio es el arbustivo y subarbustivo, lo cual podría influenciar la composición química del suelo. Debido a que en comparación con el suelo del MDM y el BE, el de este sitio con diferencias en concentración de $\mathrm{N}$ inorgánico, $\mathrm{P}, \mathrm{Fe}$ y Ca. Como muestra Schlesinger (1998), la distribución de N, P y K en el suelo está fuertemente asociada con la presencia de arbustos en hábitats desérticos. Los arbustos concentran el ciclo biogeoquímico de estos elementos en 'islas de fertilidad' bajo su dosel, mientras que en espacios estériles adyacentes están comparativamente desprovistos de actividad biótica como refiere Sánchez-González (2007).

\section{Análisis de correspondencia canónica}

En relación al ACC, las raíces características para los dos primeros ejes de ordenación (Cuadro 5) sugieren que la composición de especies entre las asociaciones vegetales, se relaciona con factores ambientales de los dos primeros ejes de variación; es decir, el análisis es satisfactorio, pues con los datos que se corresponden en los dos primeros ejes de variación se puede explicar la distribución observada y posiblemente, las características fisicoquímicas son suficientes para explicarla (Granados et al., 2013).

Cuadro 5. Análisis de correspondencia canónica con los valores de las raíces características, correlación especies-factores ambientales y porcentaje de varianza explicada por los datos de especies y relaciones especie-ambiente, para los ejes de ordenación.

\begin{tabular}{ccccc}
\hline Ejes & 1 & 2 & 3 & 4 \\
\hline Raíces características & 0.403 & 0.32 & - & - \\
Correlación especies-factores ambientales & 1 & 1 & - & - \\
Varianza acumulada (\%) de las especies & 55.8 & 100 & - & - \\
Relaciones especies-ambiente & 55.8 & 100 & - & - \\
\hline
\end{tabular}

El que la porosidad del suelo juega un rol clave en la distribución de la vegetación, también lo observó junto con altitud, pendiente y orientación, como las más relevantes en su ordenación en la SC. La clasificación de comunidades obtenida en el AC se confirma con el diagrama del ACC (Figura 5), ya que las asociaciones de los diagramas (Figuras 2 y 3) concuerdan en su ordenación. 


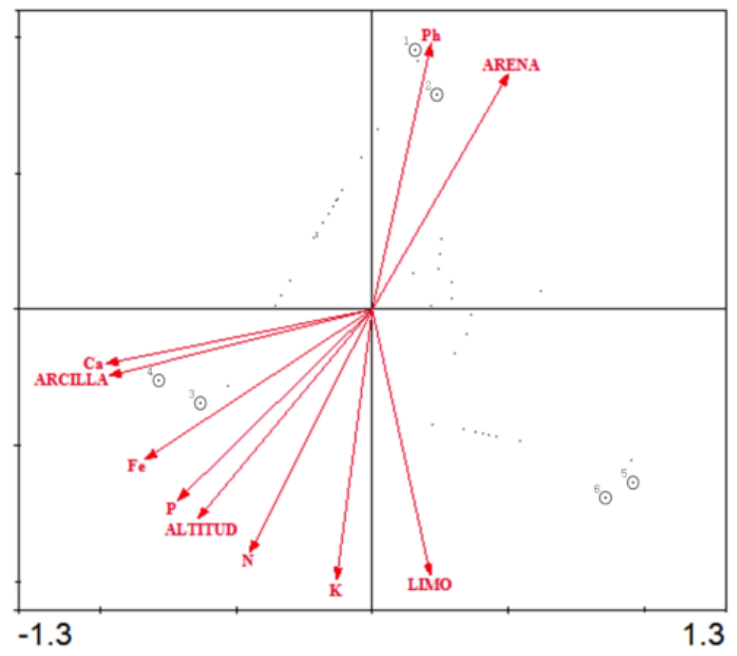

Figura 4. ACC de los transectos en el municipio de Catorce, SLP. Se incluyeron 78 especies y 10 variables ambientales. 1 y 2 (MDM), 3 y 4 (MDR) y 5 y 6 (BE).

\section{Usos de las plantas}

El estrato dominante es el arbustivo y subarbustivo (56\%). Los usos principales de las especies identificadas son medicinales $(25 \%)$, forraje $(21 \%)$ y alimento $(12 \%)$.

\section{Conclusiones}

El uso de técnicas de análisis multivariado contribuyó a la ordenación de tres comunidades vegetales, a partir de 78 especies distribuidas en el gradiente ambiental estudiado. De las variables contempladas, el $\mathrm{pH}, \mathrm{K}, \mathrm{Ca}, \mathrm{Fe}$ y altitud fueron los factores físicos que explican en mayor grado la distribución observada en las sierras y llanuras occidentales del municipio de Catorce, estos factores indirectos se relacionan con factores directos, como la humedad y temperatura, los cuales determinan la disponibilidad de agua para las plantas.

Estos sitios ostentan un alto grado de especies medicinales (principal uso detectado), endémicas, así como especies en riesgo, con información para la comprensión de este sistema ecológico que brinda el servicio ambiental de conservación in situ de acervos genéticos endémicos domesticados o silvestres y que resguarda una gran herencia biocultural bajo estatus de 'Sitio Sagrado Natural'.

\section{Literatura citada}

Braak, T. 1998. CANOCO Reference manual and user's guide to Canoco for Windows: software for canonical community ordination (version 4). Centere for Biometry. USA. 351 p.

Brush, G. S.; Lenk, C. and Smith, J. 1980. The natural forests of Maryland: an explanation of the vegetation map of Maryland. Ecological Monographs. 50(1):77-92.

CONANP. 2012. Comisión Nacional de Áreas Naturales Protegidas. Estudio previo justificativo para el establecimiento del área natural protegida de competencia de la federación con la categoría de Reserva de la Biosfera 'Wirikuta', en el estado de San Luis Potosí. SEMARNAT. México, DF. 32-33, 34-35, 48-49 pp. 
Cornelius, J. M.; Kemp; P. R.; Ludwig, J. A. y Cunningham, G. L. 1991. The distribution of vascular plant species and guilds in space and time along a desert gradient. J. Veg. Sci. 2(1):59-72.

De la Cruz, G. A. 1991. Sistema para el análisis de comunidades (ANACOM) Versión 3 (Software). Centro de Investigación y Estudios Avanzados-IPN-Unidad Mérida. Yucatán.

Gonzáles, A. 2003. Clasificación y ordenación de la vegetación del norte de la Sierra Nevada, a lo largo de un gradiente altitudinal. Anales del instituto de Biología. Serie Botánica. 74(1):47-71.

González-Costilla, O. 2007. Flora vascular de la Sierra de Catorce y territorios adyacentes, San Luis Potosí, México. Acta Botánica Mexicana. 78:1-38.

Granados, S. D. 2012. Ecología de los desiertos del mundo. Universidad Autónoma Chapingo. México. 503 p.

Granados, V. R. L.; Granados, S. D. y Sánchez, G. A. 2013. Clasificación de los bosques de pino piñonero (Pinus cembroides ssp. orizabensis) de la cuenca oriental (Puebla, Tlaxcala y Veracruz). Madera y Bosques. 2(21):23-42.

Hernández, H. 1994. Contribución al conocimiento de las cactáceas mexicanas amenazada. Acta Botánica Mexicana. 26:33-52.

Hernández, H. 2004. Checklist of Chihuahuan Desert Cactaceae. Harvard Papers in Botany. 9(1):51-68.

Herrera-Arreola, G.; Herrera, Y.; Reyes-Reyes, B. G. and Dendooven, L. 2007. Mesquite (Prosopis juliflora (Sw.) DC.), huisache (Acacia farnesiana (L.) Willd.) and catclaw (Mimosa biuncifera Benth.) and their effect on dynamics of carbon and nitrogen in soils of the semiarid highlands of Durango Mexico. J. Arid Environ. 69(4):583-598.

Hunt, D. 1999. Cactaceae checklist. Royal Botanic Gardens. International Organization of Succulent Plant Study. England. 315 p.

Jiménez, S. C. L. 2011. Las cactáceas mexicanas y los riesgos que enfrentan. Revista Digital Universitaria. 1(12):1-23.

Marroquín, J.; Borja, L. G.; Velázquez, C. R. y de la Cruz, C. J. A. 1964. Estudio ecológico dasonómico de las zonas áridas del norte de México. INIFAP. Texcoco, México. 166 p.

Montaña, C. 1990. A floristic-structural gradient related to land forms in the southern Chihuahuan Desert. J. Veg. Sci. 1(5):669-674.

Pavón, H. N. P. 2000. Distribution of plant life forms along an altitudinal gradient in the semi-arid valley of Zapotitlán, Mexico. J. Veg. Sci. 11(1):39-42.

Reyes-Reyes, B. G.; Zamora-Villafranco, E.; Reyes-Reyes, M. L.; Frias-Hernandez, J. T.; OlaldePortugal, V. and Dendooven, L. 2003. Decomposition of leaves of huisache (Acacia tortuoso) and mesquite (Prosopis spp.) in soil of the central highlands of Mexico. Plant and Soil. 256(2):359-370.

Rocha, R. 2006. Comunidades: Métodos de Estudio. Facultad de Estudios Superiores de Iztacala, Universidad Nacional Autónoma de México (UNAM). México, DF. 256 p.

Rzedowski, J. 1994. Vegetación de México. $1^{\text {ra. }}$ Edición. Limusa. México, DF. 432 p.

Sánchez-González, A. 2007. Ordenación de la vegetación de la Sierra de Catorce, San Luis Potosí, a lo largo de gradientes ambientales. Terra Latinoam. 21(3):311-319.

Schlesinger, W. 1998. Plant-soil interactions in deserts. Biogeochemistry. 1(42):169-187.

SEDUVOP. 2006. Desarrollo urbano, vivienda y obras públicas. Plan Municipal de Desarrollo Urbano de Catorce, SLP. SEDUVOP. México. 395 p.

Villarreal, J. 2017. El elemento endémico de la flora vascular del Desierto Chihuahuense. Acta Botánica Mexicana. 118:65-96. 